\title{
A case of acute lymphoblastic leukemia mimicking juvenile idiopathic arthritis
}

\author{
Derhat Demir, (D) Nilgun Eroglu, (1) Aysenur Bahadir, (1) Mukaddes Kalyoncu \\ ${ }^{1}$ Department of Pediatric Rheumatology, Karadeniz Technical University Faculty of Medicine, Trabzon, Turkey \\ ${ }^{2}$ Department of Pediatric Hematology and Oncology, Karadeniz Technical University Faculty of Medicine, Trabzon, Turkey
}

\begin{abstract}
Patients with leukemia can be presented with monoarthritis without any hematologic abnormalities. These patients may be misdiagnosed with juvenile idiopathic arthritis (JIA), and the main treatment can also be delayed. An 11-year-old girl was admitted to our pediatric rheumatology outpatient clinic with a 4-week history of swelling in the left ankle. JIA was considered as a preliminary diagnosis after the antinuclear antibody was found to be positive, and non-steroidal anti-inflammatory drug was started. Diffuse bony edema was observed in the talus, navicular, cuboid, and cuneiform bones in magnetic resonance imaging of the left ankle. Despite the treatments, the patient's joint pain increased. There were no abnormalities in repeated peripheral blood smears. On week 3 of follow-up, after bicytopenia was revealed in complete blood count, bone marrow biopsy was performed, and she was diagnosed with precursor B cell acute lymphoblastic leukemia. We presented this case to emphasize that malignancies must be evaluated in the differential diagnosis of patients with arthritis.
\end{abstract}

Keywords: Antinuclear antibodies; juvenile idiopathic arthritis; leukemia.

Cite this article as: Demir F, Eroglu N, Bahadir A, Kalyoncu M. A case of acute lymphoblastic leukemia mimicking juvenile idiopathic arthritis. North Clin Istanb 2019;6(2):184-188.

C Thildhood malignancies, without any hematologic findings, may be referred to polyclinics of pediatric rheumatology with joint complaints and may be diagnosed as juvenile idiopathic arthritis (JIA). At the time of diagnosis of malignancy, accompanying complaints of musculoskeletal system are seen in $15 \%-30 \%$ of the patients [1]. In patients with chronic arthritis, rheumatologic diagnoses are considered, and diagnosis and treatment of malignancy may be delayed. In addition, steroid treatment initiated with consideration of rheumatologic indications may mask the clinical and laboratory findings and delay the diagnosis affecting the response to chemotherapy to be given after the diagnosis [2]. We report a case of acute lymphoblastic leukemia (ALL).

Here, we present a patient with ALL who was followed up with the initial diagnosis of JIA based on the results of fine-needle aspiration biopsy performed upon the development of bicytopenia as revealed by whole blood cell count.

\section{CASE REPORT}

An 11-year-old girl without any previous health problems was admitted to our pediatric rheumatology outpatient clinic with a complaint of swelling in her left foot and ankle for 4 weeks. There were no constitutional symptoms, such as lassitude, fatigue, weight loss, night sweats, and fever. Her medical history revealed that she had swelling in the same joint lasting for 2 days that regressed with antibiotic treatment approximately 5 months ago. On physical examination, swelling in the left ankle and foot, pain, and limited range of motion of the tibiotalar and intertarsal joints were seen. Other joint and system examinations were normal. Lymphadenomegaly and hep-

Received: December 06, 2017 Accepted: March 18, 2018 Online: May 23, 2019

Correspondence: Dr. Ferhat DEMIR. Karadeniz Teknik Universitesi Tip Fakultesi, Cocuk Romatolojisi Bilim Dali, Ortahisar 61000, Trabzon, Turkey.

Phone: +90 4623775435 e-mail: drferhat@outlook.com

(c) Copyright 2019 by Istanbul Provincial Directorate of Health - Available online at www.northclinist.com

(c) (1) (8) 
atosplenomegaly were not found.

Some remarkable laboratory examination results were as follows: hemoglobin, $14.3 \mathrm{~g} / \mathrm{dL}$; white blood cell (WBC) count, $7300 / \mathrm{mm}^{3}$; absolute neutrophil count, $2500 / \mathrm{mm}^{3}$; platelet count, $419,000 / \mathrm{mm}^{3}$; erythrocyte sedimentation rate, $45 \mathrm{~mm} / \mathrm{h}$; C-reactive protein, 5.72 $\mathrm{mg} / \mathrm{dL}(\mathrm{n}=<0.5)$; and lactate dehydrogenase (LDH), $220 \mathrm{U} / \mathrm{L}(\mathrm{n}=<248)$. In peripheral blood smear, 35\% polymorphonuclear leukocytes, $60 \%$ lymphocytes, $3 \%$ monocytes, and $2 \%$ eosinophils were observed without any atypical cells. The viral and bacterial serological tests performed to reveal the etiology yielded negative results. Direct joint radiographs of both feet were within normal limits. Upon detection of antinuclear antibody (ANA) positivity (1/320-1/1000 titer, dense fine speckled pattern), naproxen sodium treatment was started with a preliminary diagnosis of monoarticular JIA, and magnetic resonance imaging (MRI) of the left foot and ankle was planned. With respect to possible uveitis, the ophthalmologic evaluation was normal. MRI revealed diffuse bone edema in the talus, navicular, cuboid, and cuneiform bones (Fig. 1). Atypical cells were not observed in recurrent peripheral blood smears performed for possible malignancy.

During follow-up, acute phase reactants were elevated, and her pain became more pronounced at night. Her arthritis did not regress in spite of the naproxen sodium treatment. On week 3 of treatment, consecutive peripheral blood smear was performed upon the presence of a WBC count of $1700 / \mathrm{mm}^{3}$, an absolute neutrophil count of $200 / \mathrm{mm}^{3}$, and a platelet count of $138,000 / \mathrm{mm}^{3}$. Atypical cells were not seen. Although atypical cells were absent in the peripheral blood smear, bone marrow aspirate was performed due to the presence of bicytopenia associated with increased bone pain at night that revealed $35 \%$ blasts, $60 \%$ lymphocytes, $4 \%$ normoblasts, and $1 \%$ myelocytes. The patient was found to be positive for CD10 and CD19 on flow cytometry and was morphologically diagnosed with pre-B cell ALL.

\section{DISCUSSION}

ALL is the most common type of cancer in childhood and constitutes $30 \%$ of childhood cancers age $<15$ years. The etiology and risk factors are not exactly known, whereas environmental, immunological, and genetic factors are thought to play a role in the onset of disease. Patients often present with fever, pallor, fatigue, lymphadenomegaly, hepatosplenomegaly, ecchymosis, and petechiae. Musculoskeletal complaints are also observed in a remarkable number of patients with leukemia before and at the time of diagnosis. Monoarthritis may be the first manifestation of malignant disease in leukemias and may lead to confusion in diagnosis $[3,4]$.

The most important step in the etiology of arthritis is to inquire the number of affected joints, the duration and pattern of the findings, the relationship with the daily activity, the previous infections, and other triggering factors during anamnesis and to evaluate the presence of a concomitant disease, if any, during an attentive physical examination. In a patient presenting with monoarthritis, septic arthritis should be the first diagnosis to be excluded if the symptoms start suddenly accompanied by fever. Local tissue infections, systemic infections (e.g., brucellosis and tuberculosis), and osteomyelitis are other causes of infection in the differential diagnosis. Mechanical causes should be evaluated first in a patient with only one joint involvement without fever. Acute rheumatic fever, systemic and polyarticular JIA, systemic lupus erythematosus, other connective tissue diseases, and vasculitis should be considered priority in patients presenting with multiple joint involvement [5]. Mechanical causes were excluded since she did not relate history of trauma and her direct radiograms were unremarkable. The presence of monoarthritis for $>4$ weeks, ANA positivity, and the continuation of the increase in acute phase reactants suggested the inflammatory causes, such as oligoarticular JIA and malignancy, in the initial diagnosis.

At the time of her first admission, patients with leukemia with musculoskeletal complaints have normal complete blood counts and peripheral blood smear at baseline and can be followed up with false rheumatologic diagnoses. There are some warning signs and symptoms for differentiation between oncologic and rheumatic diseases. In a study comparing patients with ALL and JIA, patients with leukemia were found to have bone pains increasing in severity at night independent from joint pain, whereas patients with JIA were found to have multiple joint involvement with morning stiffness, swelling, and mild pain. When peripheral blood smears were evaluated, neutrophils were predominant in patients with JIA, whereas lymphocytes in patients with leukemia [6]. Barbosa et al. reported that musculoskeletal complaints are present in $62 \%$, and that arthritis, frequently oligoarticular arthritis, are present in $13 \%$ of the cases [4].

In the same research study, seven patients were followed up with different rheumatologic and infectious 

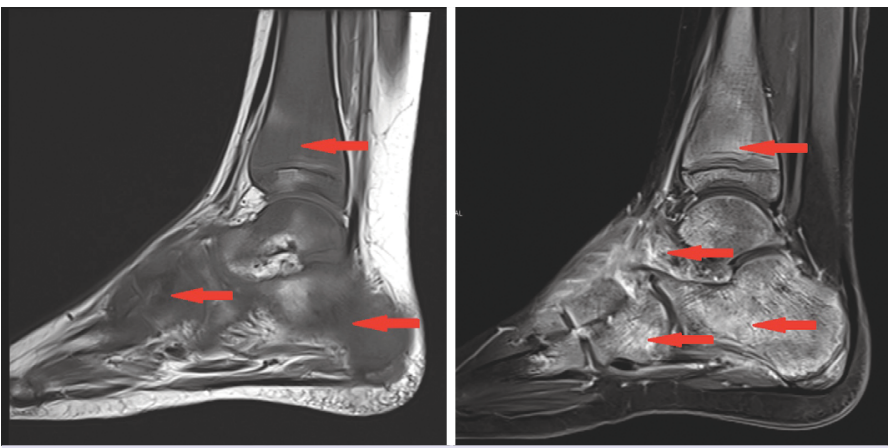

FIGURE 1. MRI of the left foot and ankle. Contrast-enhanced T1-weighted sagittal image (left) shows diffuse decreased heterogeneous signals in bone tissues and diffuse contrast enhancement in all foot bones in the fat-suppressed sagittal image (right).

diagnoses, and three cases were diagnosed with leukemia while they were receiving steroid therapy. In another recent study, of the 250 patients with preliminary rheumatologic diagnoses (60\% of whom had arthritis), 5 (2\%) were diagnosed with malignancy at follow-up. The duration of diagnosis of these patients changed between 7 days and 3 months. In patients diagnosed with malignancy, especially in the long bones, pain at night, weight loss, and fatigue were the most common complaints at admission, and any autoantibody positivity was not observed [7].

In a study comparing the clinical and laboratory findings of patients with ALL and JIA, Jones et al. reported that in patients with ALL, muscle and joint pains increase especially at night and elevated LDH and uric acid are more common, and they indicated that these findings may be useful to deviate from rheumatologic diagnoses [8]

McKay et al. reported that the diagnosis of ALL was made in a 10-year-old patient 5 months after the initial diagnosis of JIA. They noted that the diagnosis should be reviewed again in patients with joint pain increasing in severity at night and unresponsive to treatment [9]. Especially, it is stated that the decrease in hematological parameters, such as leukocyte and platelet counts, and more than two-fold increase in LDH values especially accompanied by night pains have higher diagnostic sensitivity for ALL $[8,9]$. Although whole blood cell counts were within normal limits, pain with increasing frequency and severity was a warning sign with respect to malignancy. Zombori et al. showed that LDH levels in patients with ALL have a 3-4-fold increase in blood LDH levels. The absence of morning stiffness was found to be a more robust warning sign with respect to malignancies. In the same study, it was stated that thrombocytopenia for leukemia and thrombocytosis for JIA have highly sensitive diagnostic parameters [10]. Jones et al. compared patients with ALL and JIA and could not find any significant difference between the groups with respect to the number of joints with arthritis and ANA positivity. One-third of the patients in the JIA group and $17 \%$ of the patients in the ALL group were found to have ANA positivity [8].

In another study where ANA positivity was investigated in patients with malignancy, 65 (19\%) out of the 342 patients in whom other potential causes of antibody positivity were excluded, antibody positivity was found to be 19 times higher than that of the control group [11]. Although the diagnostic sensitivity of ANA is known in autoimmune diseases, these findings demonstrate that it is not very useful with respect to distancing from malignancies. Our patient presented with monoarthritis and her night pain became evident in her follow-up, whereas her peripheral blood smear revealed lymphocyte dominance. Non-steroidal anti-inflammatory treatment was initiated in our patient who showed similarities with warning signs in the above-mentioned studies, and malignancies were kept in mind in the differential diagnosis. In the follow-up, frequent peripheral blood smears were performed due to the development of bicytopenia and widespread bone edema seen in MRI. Although no blast was seen in all peripheral blood smears, bone marrow aspiration was performed to diagnose ALL.

Peripheral blood smear evaluation plays an important role in the diagnosis of ALL. Atypical cells in peripheral smear can warn physicians for the presence of malignancy, although any abnormality in whole blood count especially in the early period is not seen. On the other hand, the absence of blast in peripheral blood smear should never lead to the exclusion of malignancies in the differential diagnosis. In different studies conducted in this regard, it has been reported that in the diagnosis of ALL, blasts may not be seen in peripheral blood smears of up to $83 \%$ of the cases $[1,6,12]$. In our case, no blasts were seen in recurrent peripheral blood smears, including the period in which bicytopenia was observed in the complete blood count, but the diagnosis could be made only after the evaluation of bone marrow aspirates.

Another examination modality that may be useful in the differential diagnosis of JIA and ALL is MRI. In T1-weighted studies, diffuse signal reduction and bone 
marrow involvement are frequently seen in patients with leukemia. In the presence of these findings, peripheral blood smear examination, bone marrow aspiration, and biopsy should be performed for definite diagnosis in consideration of malignancy. In patients with JIA, findings of synovitis, tenosynovitis, and enthesitis in MRI were significant in the diagnosis of the disease, but the diagnostic value of bone edema could not be determined.

Although diffuse involvement of more than one bone is seen in ALL, local bone marrow edema can be frequently seen in the vicinity of synovitis in JIA. The presence of enthesitis and tenosynovitis was evaluated as findings that could help to remove the physician away from the possibility of malignancies in the differential diagnosis $[13,14]$. Tsujioka et al. compared patients with leukemia with JIA and osteoarthralgia, and MRIs of painful joints of three patients demonstrated bone marrow edema similar to osteomyelitis in two patients and periarticular synovitis-like inflammation in one patient. In the same study, the patient with periarticular synovitis-like inflammation in the MRI was diagnosed with ALL after blast cells were in the peripheral blood smear during follow-up [15].

This finding shows that bone marrow edema is a more specific MRI finding for ALL, but synovial involvement cannot exclude the diagnosis. However, in patients with bone marrow edema on MRI, non-bacterial osteomyelitis should not be ignored in the differential diagnosis. In our patient who was monitored with monoarthritis and ANA positivity, MRI revealed diffuse bone edema in all foot bones and diffuse signal reduction in T1-weighted images. Delays in the diagnosis and treatment of oncological diseases may have more dramatic results than other disease groups. In addition, steroid and cytotoxic treatments after misdiagnosis may affect the response of patients to the treatment of malignancy.

Révész et al. [2] determined that 34 out of 699 patients who were diagnosed and followed up with ALL had previously received different doses of steroid treatment due to aplastic events or arthritis-based rheumatic diseases, and so they remained in remission for a short time after the application of chemotherapy.

Our patient was followed up with naproxen sodium treatment because clinical and laboratory tests were initially compatible with JIA. After the appearance of hematological findings, she was diagnosed with ALL, and chemotherapy was started.

In conclusion, patients who presented with muscu- loskeletal system symptoms and who were diagnosed with malignant disease may be followed up with preliminary diagnoses, such as JIA, reactive arthritis, infectious arthritis, or growth pain. These patients may experience delays in their actual diagnosis and treatment and may face with adverse outcomes of the disease in the future. We presented this case to draw attention to the fact that malignancy should be kept in mind at all times in patients with JIA, even though complete blood count and peripheral blood smear results are within normal limits. In patients with non-typical JIA and monoarthritis accompanying with intense night pain, highly elevated acute phase reactants, and bone edema in MRI, we believe that bone marrow aspiration should be performed before treatment even if ANA positivity is present.

Informed Consent: Written informed consent was obtained from the parents of the patient for publication of this case report and accompanying images.

Conflict of Interest: The authors declare that there are no conflicts of interest.

Financial Disclosure: The authors declared that this study has received no financial support.

Authorship Contributions: Concept - FD, NE, AB, MK; Design FD, MK; Supervision - AB, MK; Materials - FD, NE; Data collection and/or processing - FD; Anaylsis and/or interpretation - FD, $A B, M K$; Writing - FD; Critical review - AB, MK.

\section{REFERENCES}

1. Cabral DA, Tucker LB. Malignancies in children who initially present with rheumatic complaints. J Pediatr 1999;134:53-7. [CrossRef]

2. Révész T, Kardos G, Kajtár P, Schuler D. The adverse effect of prolonged prednisolone pretreatment in children with acute lymphoblastic leukemia. Cancer 1985;55:1637-40. [CrossRef]

3. Silverman LB. Acute lymphoblastic leukemia. In: Orkin SH, Fisher DE, Lux SE, Ginsburg D, Nathan DG, editors. Oncology of Infancy and Childhood. Philadelphia: Elsevier; 2009. p. 297-330. [CrossRef]

4. Barbosa CM, Nakamura C, Terreri MT, Lee ML, Petrilli AS, Hilário MO. Musculoskeletal manifestations as the onset of acute leukemias in childhood. [Article in Portuguese]. J Pediatr (Rio J) 2002;78:481-4.

5. Kasapçopur Ö. Çocukluk Çağı Romatizmal Hastalıklarına Tanılandırıcı Yaklaşım. Romatoloji Hastalıkları Sempozyum Dizisi 2003;34:43-50.

6. Ostrov BE, Goldsmith DP, Athreya BH. Differentiation of systemic juvenile rheumatoid arthritis from acute leukemia near the onset of disease. J Pediatr 1993;122:595-8. [CrossRef]

7. Fonseca MB, Gomes FHR, Valera ET, Pileggi GS, Gonfiantini PB, Gonfiantini $\mathrm{MB}$, et al. Signs and symptoms of rheumatic diseases as first manifestation of pediatric cancer: diagnosis and prognosis implications. Rev Bras Reumatol Engl Ed 2017;57:330-7. [CrossRef]

8. Jones OY, Spencer CH, Bowyer SL, Dent PB, Gottlieb BS, Rabinovich CE. A multicenter case-control study on predictive factors distinguishing childhood leukemia from juvenile rheumatoid arthritis. Pediatrics 
2006;117:e840-4. [CrossRef]

9. McKay D, Adams L, Ostring G, Singh-Grewal D. In a child presenting with features consistent with a diagnosis of juvenile idiopathic arthritis, what clinical features or laboratory findings (at presentation) predict a diagnosis of acute lymphoblastic leukaemia? J Paediatr Child Health 2010;46:442-5. [CrossRef]

10. Zombori L, Kovacs G, Csoka M, Derfalvi B. Rheumatic symptoms in childhood leukaemia and lymphoma-a ten-year retrospective study. Pediatr Rheumatol Online J 2013;11:20. [CrossRef]

11. Burnham TK. Antinuclear antibodies in patients with malignancies. Lancet 1972;2:436. [CrossRef]

12. Brix N, Rosthøj S, Herlin T, Hasle H. Arthritis as presenting manifes- tation of acute lymphoblastic leukaemia in children. Arch Dis Child 2015;100:821-5. [CrossRef]

13. Silva JR Jr, Hayashi D, Yonenaga T, Fukuda K, Genant HK, Lin C, et al. MRI of bone marrow abnormalities in hematological malignancies. Diagn Interv Radiol 2013;19:393-9. [CrossRef]

14. Breton S, Jousse-Joulin S, Finel E, Marhadour T, Colin D, de Parscau L, et al. Imaging approaches for evaluating peripheral joint abnormalities in juvenile idiopathic arthritis. Semin Arthritis Rheum 2012;41:698-711.

15. Tsujioka T, Sugiyama M, Ueki M, Tozawa Y, Takezaki S, Ohshima J et al. Difficulty in the diagnosis of bone and joint pain associated with pediatric acute leukemia; comparison with juvenile idiopathic arthritis. Mod Rheumatol 2017;14:1-6. [CrossRef] 